\title{
Bestandserhaltung mit System
}

Bestände-Priorisierung und Schadenserfassung

Über sechs Millionen Medieneinheiten stehen im Bestand der Württembergischen Landesbibliothek und machen sie zur größten wissenschaftlichen $\mathrm{Bi}$ bliothek in Baden-Württemberg. Zu den bibliothekarischen Aufgaben gehören das Sammeln, ErschlieBen und Bereitstellen - aber auch das Aufbewahren und die Erhaltung der Bestände.

Schriftliche Dokumente erfordern aufgrund ihrer Materialität den Schutz vor Schäden durch äußere Faktoren wie Schmutz und Wasser, Schimmel, Schädlinge oder Benutzung, um sie langfristig zu erhalten. Chemische Zersetzungsprozesse zerstören zusätzlich ihre Substanz, wie man am Beispiel von Tintenfraß oder säurehaltigem und dadurch brüchigem Papier deutlich sehen kann.

Doch wie geht man bei der Bestandserhaltung angesichts von solchen Mengen bedeutenden Kulturgutes vor? Woher wissen wir, welche Bestände beschädigt sind - und vor allem: Wie hoch ist das Ausmaß und welche Maßnahmen müssen dringend durchgeführt werden, um weitere Schäden zu verhindern und diese reichen Sammlungen dauerhaft für unsere Nachwelt zu erhalten?

Bereits 2001 schlossen sich mehrere Archive und Bibliotheken zusammen und bildeten die "Allianz zur Erhaltung des schriftlichen Kulturguts". Die Aufmerksamkeit wurde darauf gelenkt, dass die Erhaltung von bedeutsamem schriftlichem Kulturgut keine Einzelaufgabe der Institutionen sein kann, sondern nur durch konzentriertes bzw. kooperativabgestimmtes Handeln und auf nationaler Ebene zu bewältigen ist.

Erstmalig wurden 2010 von Bund und Ländern Mittel zur Verfügung gestellt, und die Politik konnte durch beispielhafte Restaurierungsmaßnahmen von einer dauerhaften Finanzierung bestandserhaltender Projekte überzeugt werden.

In diesem Jahr feiert die Koordinierungsstelle für die Erhaltung schriftlichen Kulturguts (KEK) ihr zehnjähriges Bestehen als nachhaltiges BundLänder-Projekt, angesiedelt an der Staatsbibliothek zu Berlin - Preußischer Kulturbesitz. Neben der Entwicklung einer nationalen Gesamtstrategie zum
Erhalt des schriftlichen Kulturguts und einer breiten Öffentlichkeitsarbeit koordiniert die KEK die Vergabe von Fördermitteln der Beauftragten der Bundesregierung für Kultur und Medien (BKM) bzw. der Kulturstiftung der Länder (KSL).

Nachdem auch die Württembergische Landesbibliothek in den letzten Jahren mehrere Projekte und Einzelrestaurierungen mit Hilfe der KEK umsetzen konnte, wurde im Herbst 2020 ein Projekt finanziert, das sich losgelöst vom Einzelobjekt auf den historischen Sonderbestand konzentrierte. Handlungsbedarf gibt es bei einer Bibliothek mit über sechs Millionen Einheiten Bibliotheksgut in allen großen und kleinen Teilbeständen, daher stellten sich die Fragen:

Wie kann eine Priorisierung mit System erfolgen - welche Bestandssegmente sind vielleicht historisch besonders wertvoll und aufgrund festgelegter Kriterien vorzuziehen? Und in welchem Gesamtzustand und Schadensausmaß befinden sich die Bestände - ist eine Schadensklassifizierung möglich, um zu entscheiden, an welcher Stelle Maßnahmen im Mengenverfahren eingeleitet werden? Lassen sich daraus die Kosten für die kommenden Jahre abschätzen?

\section{Pilotprojekt zur Priorisierung und Schadenser- fassung gefährdeter Bestände der Sondersamm- lungen der WLB als Vorbereitung zur Durchfüh- rung von Maßnahmen des Originalerhalts}

\footnotetext{
Ausgangspunkt des Projektes war die strategische Notwendigkeit, zu einer fundierten und systematischen Planung von Maßnahmen der Bestandserhaltung im Mengenverfahren zu kommen.

Eine Schadenserfassung an allen Sondersammlungen durchzuführen, erschien angesichts der großen Bestandsvolumina von vornherein unrealistisch. Daher wurden repräsentative Teilbestände aus den Sondersammlungen der WLB ausgewählt und anhand bestimmter Kriterien priorisiert. Diese sollten anschließend einer systematischen Schadensanalyse unterzogen werden, um Rückschlüsse zum
} 
Erhaltungszustand und Schadensausmaß ziehen zu können. Solche statistischen Ergebnisse lassen sich wiederum auf den Gesamtbestand übertragen und ermöglichen es dadurch, den künftigen Bedarf bestandserhaltender Maßnahmen abzuschätzen.

\section{Beständepriorisierung durch die Sammlungsleiter}

Um die Bestände untereinander priorisieren zu können (jedes Objekt hat zweifelsohne seinen eigenen intrinsischen Wert), identifizierten die Sammlungsleiter anhand eines Kriterienkatalogs besonders gefährdete und zugleich repräsentative Teilbestände aus ihren Sondersammlungen. Neben den grundlegenden, allgemeinen Angaben (z.B. Signaturengruppe, Bestandstyp oder Umfang), galt es folgende Kriterien einzustufen: Rarität bzw. Unikalität, Alleinstellungsmerkmal bzw. nationale/ internationale Bedeutung, Benutzungsfrequenz, Erschließungsgrad sowie eine erste, grobe Einschätzung zum Umfang des Maßnahmenbedarfs und der bereits durchgeführten Maßnahmen.

Die Auswertung erfolgte über einen Gewichtungsschlüssel, der alle Angaben miteinander verrechnete und dadurch eine methodisch plausible Übersicht der Teilbestände ergab. Die Priorisierung brachte so eine Positionierung in der Reihenfolge der Teilsammlungen mit sich.

Das Projektvolumen ermöglichte eine Bearbeitung von insgesamt ca. 10.000 Bibliothekseinheiten: eine Bearbeitungsmenge, die zum einen finanzierbar und zum anderen vom Dienstleister im festgesetzten Zeitraum zu bewältigen war - schließlich muss bei einer Schadenserfassung jedes einzelne Objekt entnommen, analysiert, erfasst und zurückgestellt werden. Um möglichst viele repräsentative Bestandssegmente analysieren zu können, wurde eine Teilerhebung mit einer Stichprobengröße von $10 \%$ ausgewählt, die bereits in Archiven erfolgreich angewendet wird.

Aus der priorisierten Liste wurden also so viele Sammlungsteile ausgewählt, dass bei der Anwendung des Stichprobenverfahrens eine Bearbeitungsmenge von insgesamt 10.000 Bibliothekseinheiten zustande kam. Somit konnten 15 Bestandssegmente für eine Schadenserfassung identifiziert werden, die zu folgenden Sondersammlungen gehören:

\begin{tabular}{|r|l|l|}
\hline & Sammlung & Teilbestand \\
\hline $\mathbf{1}$ & Alte und Wertvolle Drucke & Lateinische Bibeln (Oktav) \\
$\mathbf{2}$ & Alte und Wertvolle Drucke & Theologischer Altbestand (Folio) \\
$\mathbf{3}$ & Alte und Wertvolle Drucke & Teile der Hofbibliothek \\
$\mathbf{4}$ & Alte und Wertvolle Drucke & Bände zur Kunst- und Architekturgeschichte im Überformat \\
$\mathbf{6}$ & Stefan George Archiv & Annotiertes Aufführungsmaterial in Großkapseln \\
$\mathbf{7}$ & Musik & Bibliothek Stefan Georges, Monographien, Separata \& Zeitungsausschnitte \\
$\mathbf{8}$ & Stefan George Archiv & Aufführungsmaterial, Einbände \\
$\mathbf{9}$ & Musik & Archivbibliothek Druckschriftensammlung, Monographien, Separata \& Rara \\
$\mathbf{1 0}$ & Bibliothek für Zeitgeschichte & Buchbestand Nachkriegszeit bis 1965 \\
$\mathbf{1 1}$ & Musik & gebundene Theaterzettel \\
$\mathbf{1 2}$ & Musik & Annotiertes Aufführungsmaterial Theater (Kapselbestand) \\
$\mathbf{1 3}$ & Musik & Libretti, Broschurensammlung \\
$\mathbf{1 4}$ & Alte und Wertvolle Drucke & Ältester Teil der Sammlung Hugo Borst \\
$\mathbf{1 5}$ & Bibliothek für Zeitgeschichte & Flugblätter, Zeit der Weltkriege \\
\hline
\end{tabular}

\section{Schadenserfassung durch restaurato- rische Fachkräfte}

Die Erfassung erfolgte direkt am Standort der Bestände in den Magazinbereichen. Ansprechpartner der Sondersammlungen hielten sich für Rückfragen bereit. Regale wurden markiert, Bücherwagen als Ablageflächen bereitgestellt, Kabeltrommeln entrollt und mobile Arbeitsplätze immer wieder neu eingerichtet. Erfasst wurden Einbände, Mappen, Kapselbestände und Schrankinhalte einmal quer durch das Magazin im zweiten Untergeschoss der Bibliothek, hoch ins Obergeschoss zum Stefan George Archiv und von dort hinüber in die Außenstelle Gaisburgstraße. 

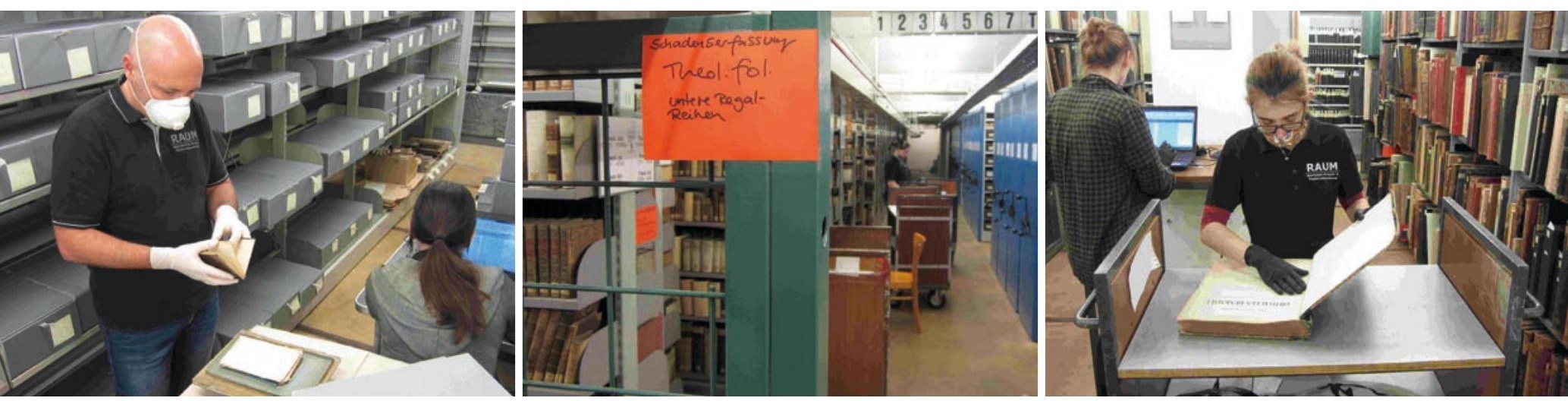

Abb. 1: Erfassung der Libretti, des theologischen Altbestandes und der überformatigen Kunstbände

Die Werkstatt für Buch- und Papierrestaurierung Matthias Raum führte diese Arbeiten mit drei Zweier-Teams über knapp zwei Wochen hinweg durch. Die systematische Stichprobenerhebung bedeutet in der Bearbeitung, dass jeder zehnte Band oder jede zehnte Kapselverpackung aus dem Regal genommen und zunächst neben der Signatur objektbezogene Daten aufgenommen wurden: Objektart (z.B. handschriftliches Material, Einband oder Loseblatt-Sammlung), Einbandmaterial, Buchblock bzw. Trägermaterial, gebundenes/ungebundenes Objekt sowie eine Einteilung in drei Formate. Die Angaben liefern bei der Auswertung entscheidende Hinweise, denn nicht jede Restaurierungsmaßnahme ist universal anwendbar. Ledereinbände können beispielsweise nicht ohne Verlust am Lederbezug in ein Entsäuerungsverfahren gegeben werden, das aber für das säurehaltige Papier des Buchblocks erforderlich sein kann.

\section{Erhaltungszustand nach definierten Schadens- klassen (SK):}

Die vorliegenden Schäden wurden anschließend von einer Fachkraft analysiert und tabellarisch von einer zweiten Fachkraft am Laptop erfasst. Jedes einzelne Objekt (bei Einzelblättern wurde mappenweise erfasst) erhielt zunächst eine Einstufung in Schadensklassen:

SK 1 - guter Zustand, Gebrauchsspuren

SK 2 - mittlerer Erhaltungszustand mit leichten

Schäden und geringem Risiko weiterer

Nutzungsschäden

SK 3 - schlechter Erhaltungszustand mit starken Schäden und eingeschränkter Nutzung in Ausnahmefällen

SK 4 - sehr schlechter Erhaltungszustand,

Objekte sollten für Nutzung gesperrt werden, unmittelbarer Substanzverlust.
Der Erhaltungszustand einer Sammlung konnte in diesem ersten Schritt abgegrenzt werden. Doch was bedeutet dieser genau? Welche Schäden liegen vor? Und welche Maßnahmen sind erforderlich, um die langfristige Erhaltung des Bestandes zu gewährleisten? Müssen leichte Schäden eher schnell behoben werden?

\section{Schadensbilder und Maßnahmenbedarf}

Um diese Fragen genauer beantworten zu können und auch die erforderlichen Maßnahmen damit zu verknüpfen, wurden im nächsten Schritt der Erfassung die genauen Schadensbilder analysiert und festgehalten: fortschreitende Schäden wie Farb- bzw. Tintenfraß und Selbstklebebänder, säurehaltiges Papier, Verblockung und Verklebung, Schimmelbefall, starke Oberflächenverschmutzung, mechanische Schäden wie große Risse oder Einbandschäden und schließlich eine unverpackte Lagerung.

Die erforderlichen Maßnahmen wurden im letzten Schritt angegeben und konzentrieren sich auf Mengenverfahren, die an großen Sammlungsteilen erfolgen: Trockenreinigung, Schutzverpackung, Mengenentsäuerung und die serielle Einzelrestaurierung von Einheiten mit strukturell homogenem Schadensbild.

Regelmäßige Besprechungen und Rückmeldungen zwischen WLB und der Dienstleister-Firma Raum klärten offene Fragen, justierten an der einen oder anderen Stelle etwas nach und brachten alle Beteiligten auf den gleichen Kenntnisstand. Dies stellte sich als unverzichtbar heraus, denn die Definition "Bibliothekseinheit" ist beispielsweise klar zu formulieren, wenn es sich um solch diverse Objekte handelt, die in diesem Projekt erfasst wurden. Zählt z.B. eine Mappe als Einheit oder jedes eingelegte 
Blatt? Wie mache ich eine erfasste Angabe nachprüfbar, wenn es nur Sammelsignaturen gibt?

So war etwa die Druckschriftensammlung des Stefan George Archivs (StGA) zum Zeitpunkt der Erfassung noch nicht vollständig mit Einzelsignaturen

\section{Risikoanalyse}

Die Auswertung der erfassten Daten erfolgte ähnlich wie bei der Priorisierung nach einem in Referenzprojekten aus dem Archivbereich bereits bewährten Schlüssel zur Gewichtung. In die Planung

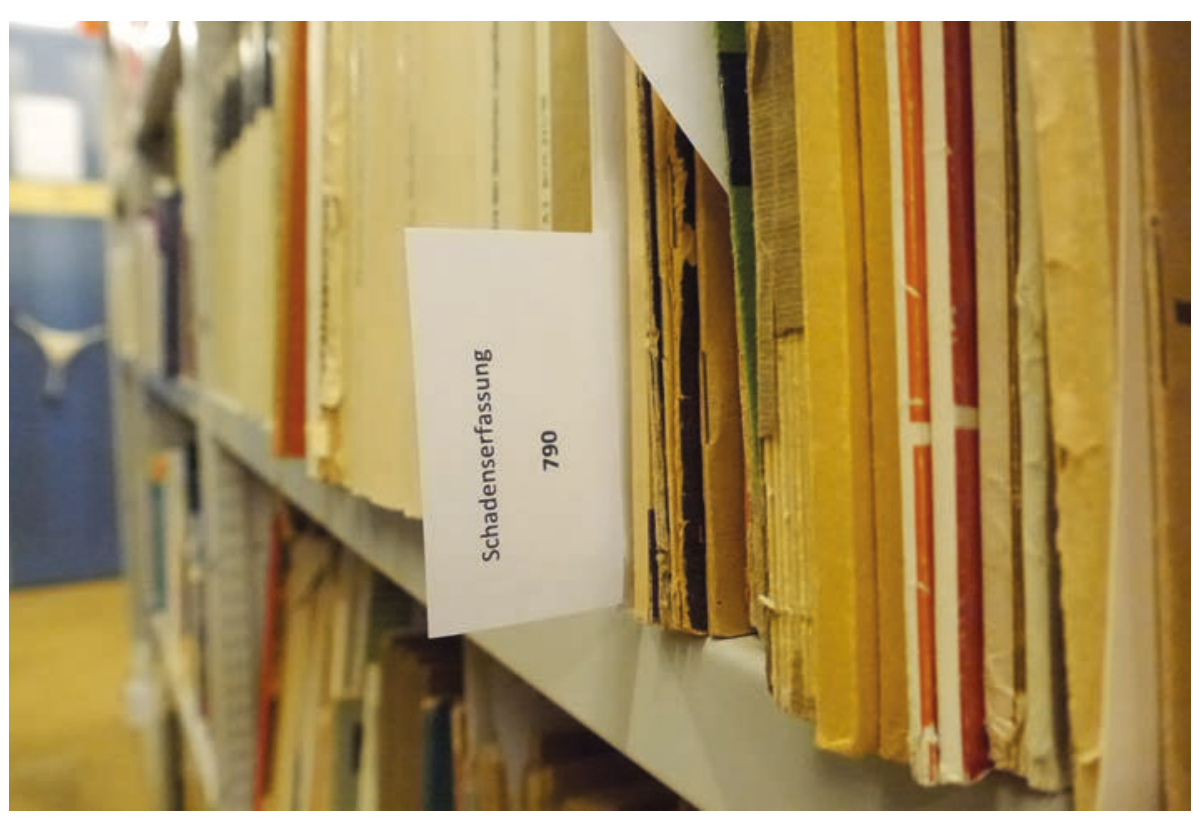

Abb. 2: Kennzeichnung der Monographien im StGA

versehen. Als pragmatische Lösung wurden daher nummerierte Streifen eingelegt, die sich später mit der Vergabe der Signaturen verknüpfen ließen. von Maßnahmen zur Bestandserhaltung sollten neben der Einschätzung der kulturgeschichtlichen Bedeutung aufgrund von Rarität, Materialität und allgemeinem Erhaltungszustand auch die differenzierten Analysen zu vorliegenden Schäden und sachgemäßen Verfahren zu deren Behandlung einbezogen und miteinander verrechnet werden.

Die Risikoanalyse erweist sich dadurch als mathematisches Produkt von Schadenserfassung und Bestände-Priorisierung. Angesichts begrenzter personeller und finanzieller Ressourcen ergibt sich auf diese Weise eine quantifizierte Analyse der Risiken im Hinblick auf einen ohne rechtzeitiges oder angemessenes Eingreifen eintretenden Bestandsverlust.

Ergebnis der Risikoanalyse durch Priorisierung und Schadenserfassung:

\begin{tabular}{|r|l|l|}
\hline & Sammlung & Teilbestand \\
\hline $\mathbf{1}$ & Musik & Libretti, Broschurensammlung \\
$\mathbf{2}$ & Musik & Aufführungsmaterial, Einbände \\
$\mathbf{3}$ & Stefan George Archiv & Bibliothek Stefan Georges \\
$\mathbf{4}$ & Musik & Annotiertes Aufführungsmaterial in Großkapseln \\
$\mathbf{5}$ & Musik & Annotiertes Aufführungsmaterial Theater (unkatalogisierter Kapselbestand) \\
$\mathbf{6}$ & Musik & gebundene Theaterzettel \\
$\mathbf{7}$ & Musik & Gesamt- und Denkmälerausgaben \\
$\mathbf{8}$ & Stefan George Archiv & Archivbibliothek Druckschriftensammlung \\
$\mathbf{9}$ & Bibliothek f. Zeitgeschichte & Flugblätter, Zeit der Weltkriege \\
$\mathbf{1 0}$ & Bibliothek f. Zeitgeschichte & Buchbestand Nachkriegszeit bis 1965 \\
$\mathbf{1 1}$ & Alte und Wertvolle Drucke & Lateinische Bibeln (Oktav) \\
$\mathbf{1 2}$ & Alte und Wertvolle Drucke & Bände zur Kunst- und Architekturgeschichte im Überformat \\
$\mathbf{1 3}$ & Alte und Wertvolle Drucke & Teile der Hofbibliothek \\
$\mathbf{1 4}$ & Alte und Wertvolle Drucke & Theologischer Altbestand (Folio) \\
$\mathbf{1 5}$ & Alte und Wertvolle Drucke & Ältester Teil der Sammlung Hugo Borst \\
\hline
\end{tabular}




\section{Maßnahmenbedarf}

In Bezug auf die Gesamtheit der stichprobenweise analysierten Bestandssegmente lässt sich ein nach prioritären Verfahren und zugleich Dringlichkeit geordneter Bedarfsumfang an Maßnahmen kalkulieren.

Trockenreinigung und Verpacken

\begin{tabular}{|c|l|l|l|r|}
\hline Prio & Sammlung & Teilbestand & Zustand & Behandlungs-Menge \\
\hline $\mathbf{2}$ & Musik & Aufführungsmaterial, Einbände & SK 1-3 & 2.340 Bände (100\%) \\
$\mathbf{3}$ & StGA & Bibliothek Stefan Georges, - Monographien & SK 1-2 & 940 Einbände (100\%) \\
& & - Separata & SK 1-3 & 748 Einheiten (88\%) \\
$\mathbf{6}$ & Musik & gebundene Theaterzettel & SK 2 & 228 Einheiten (100\%) \\
$\mathbf{7}$ & Musik & Gesamt-/Denkmälerausgaben & SK 1-2 & 1.700 Einheiten (100\%) \\
$\mathbf{8}$ & StGA & Archivbibliothek Druckschriftensammlung & & \\
& & - Rara & SK 1 & 272 Einheiten (80\%) \\
& & - Monographien & SK 1-2 & 2.408 Verpacken (54\%) \\
$\mathbf{1 0}$ & BfZ & Buchbestand Nachkriegszeit & SK 1 & 39.666 Einheiten (100\%) \\
$\mathbf{1 1}$ & AD & Lateinische Bibeln (Oktav) & SK 2 & 770 Einheiten (100\%) \\
$\mathbf{1 2}$ & AD & Kunst- und Architekturgeschichte, Überformat & SK 1-2 & 1.000 Einheiten (100\%) \\
$\mathbf{1 3}$ & AD & Teile der Hofbibliothek & SK 1-2 & 6.000 Einheiten (100\%) \\
$\mathbf{1 4}$ & AD & Theologischer Altbestand (Folio) & SK 1-2 & 2.200 Einheiten (100\%) \\
$\mathbf{1 5}$ & AD & Ältester Teil der Sammlung Hugo Borst & Sk 1-2 & $2.000(100 \%)$ \\
& & & & $=\mathbf{6 0 . 2 9 2 ~ E i n h e i t e n ~}$ \\
\hline
\end{tabular}

Mengenentsäuerung von säurehaltigen Papieren

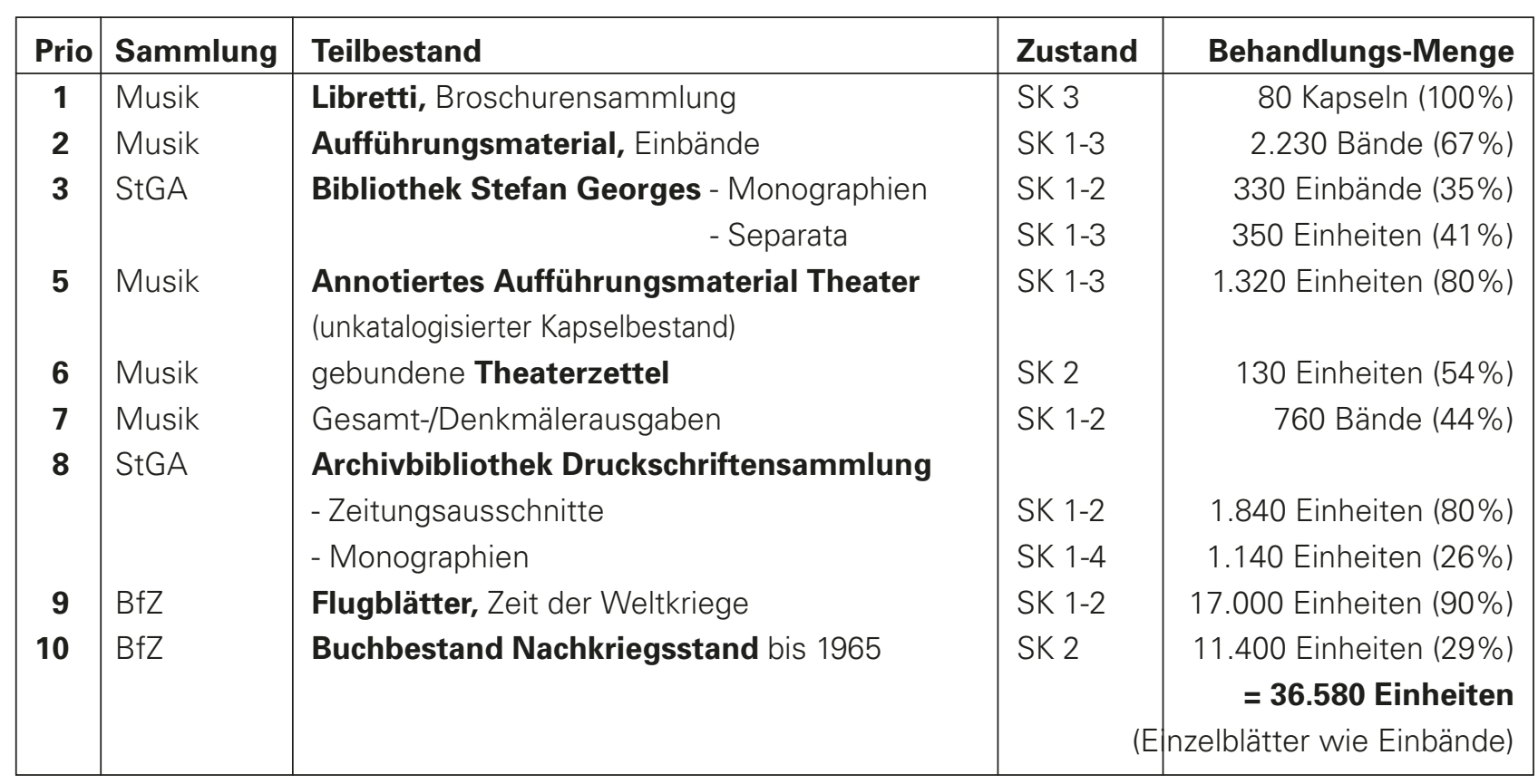




\section{Serielle Restaurierungs- und Stabilisierungsmaßnahmen im Mengenverfahren}

Entfernen von Klebebändern, Papierstabilisierung von Rissen usw.

\begin{tabular}{|c|c|c|c|c|}
\hline Prio & Sammlung & Teilbestand & Zustand & Behandlungs-Menge \\
\hline 1 & Musik & Libretti, Broschurensammlung & SK 3 & 40 Kapseln (50\%) \\
\hline 2 & Musik & Aufführungsmaterial, Einbände & SK $1-3$ & 2.340 Bände (100\%) \\
\hline 3 & StGA & $\begin{array}{c}\text { Bibliothek Stefan Georges - Monographien } \\
\text { - Separata }\end{array}$ & $\begin{array}{l}\text { SK } 4 \\
\text { SK } 1-3\end{array}$ & $\begin{array}{l}395 \text { Einbände ( } 42 \%) \\
442 \text { Einheiten }(52 \%)\end{array}$ \\
\hline 5 & Musik & $\begin{array}{l}\text { Annotiertes Aufführungsmaterial } \\
\text { in Großkapseln }\end{array}$ & SK $1-2$ & 25 Kapseln (8\%) \\
\hline 5 & Musik & $\begin{array}{l}\text { Annotiertes Aufführungsmaterial Theater } \\
\text { (unkatalogisierter Kapselbestand) }\end{array}$ & $\begin{array}{l}\text { SK } 1-3 \\
\text { SK } 1-2\end{array}$ & $\begin{array}{l}\text { 1.132 Einheiten (69\%) } \\
\text { 2.408 Verpacken (54\%) }\end{array}$ \\
\hline 6 & Musik & gebundene Theaterzettel & SK 2 & 48 Bände (21\%) \\
\hline 7 & Musik & Gesamt-/Denkmälerausgaben & SK $1-2$ & 1.700 Bände (100\%) \\
\hline 8 & StGA & Archivbibliothek Druckschriftensammlung & & \\
\hline & & $\begin{array}{l}\text { - Zeitungsausschnitte } \\
\text { - Rara }\end{array}$ & $\begin{array}{l}\text { SK } 1-2 \\
\text { SK } 1-2\end{array}$ & $\begin{array}{l}547 \text { Einheiten (24\%) } \\
109 \text { Einheiten (32\%) }\end{array}$ \\
\hline 9 & $\mathrm{BfZ}$ & Flugblätter, Zeit der Weltkriege & SK $1-2$ & 7.200 Einheiten (36\%) \\
\hline 10 & $\mathrm{BfZ}$ & Buchbestand Nachkriegszeit bis 1965 & SK 2 & $\begin{array}{r}20 \text { Einheiten }(0,5 \%) \\
=\mathbf{1 3 . 9 9 8} \text { Einheiten } \\
\text { (Einzelblätter wie Einbände) }\end{array}$ \\
\hline
\end{tabular}

Aufgrund jeweils anzufragender Angebote von Dienstleistern kann daraus eine ungefähre Kostenschätzung und ein Mittelbedarf für Bestandserhaltungsmaßnahmen abgeleitet werden.

Das Stichprobenverfahren und der exemplarischrepräsentative Charakter der in der Vorauswahl bestimmten Bestandssegmente lassen längerfristig ausgerichtete Prognosen zur Schadenssituation des Gesamtbestandes der Sondersammlungen zu. Die hier analysierten Teilbestände stehen jeweils für ein größeres Ganzes, das sich in einem strukturell vergleichbaren Zustand befindet. Aber bereits der Umfang der in diesem Projekt analysierten Teilsammlungen macht eine vieljährige Projektplanung notwendig.

Der aktuell vielleicht größte Handlungsbedarf besteht mit knapp über 60.000 Bibliothekseinheiten im Bereich der Trockenreinigung und Schutzverpackung. Gerade im Hinblick auf die Umzugsvorhaben und Lagerungssituationen der Bestände an verschiedenen Standorten konzentrieren sich die geplanten Folgeprojekte für 2021 daher auf diese Maßnahmen.

Von gleicher Relevanz zeigt sich der Bedarf an Mengenentsäuerungen. Die verzeichneten Erhaltungszustände der Papiere liegen bisher überwiegend

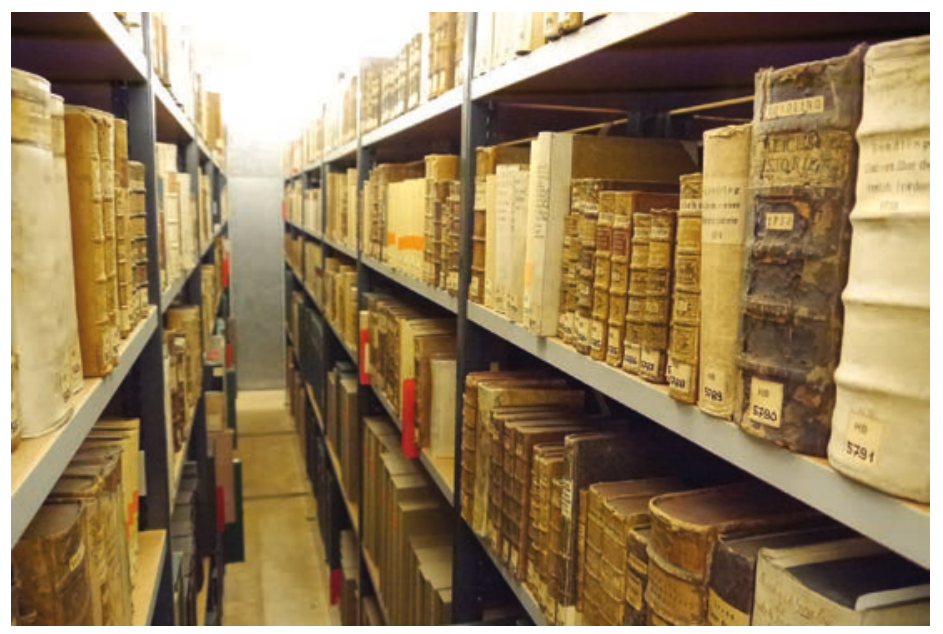

Abb. 3: Bände der Hofbibliothek aus der Sammlung Alte und Wertvolle Drucke

in den Schadensklassen 1-2 und bilden daher gute Voraussetzungen für eine erfolgreiche und nachhaltige Behandlung (stark versprödete Papiere profitieren beispielsweise nur noch sehr bedingt von einer Entsäuerung).

Die Notwendigkeit der seriellen Stabilisierungsmaßnahmen wurde an 10 Teilbeständen ermittelt und macht zwei Drittel der gesichteten Bestandsgruppen aus. Solche Mengenverfahren stehen teilweise noch in der Entwicklung und erfordern neben einer anderen Herangehensweise als bei Einzelrestaurierungen (wie sie im Buchpatenschaftsprogramm 
angewendet werden) eine komplexere Logistik und Vorbereitung.

Insgesamt wurde eine auch für andere Bibliotheken modellhafte Vorgehensweise gefunden, um ein mittel- und längerfristig angelegtes Arbeitsprogramm einer empirisch gestützten und systematisch agierenden Bestandserhaltung zu entwickeln.
Für die nächsten Jahre sind die anzustrebenden Maßnahmen der WLB durch die Schadenserfassung nun systematisch beleuchtet worden - sie beziehen sich auf präventive, konservatorische und restauratorische Maßnahmen gleichermaßen.

Sonja Brandt / Christian Herrmann

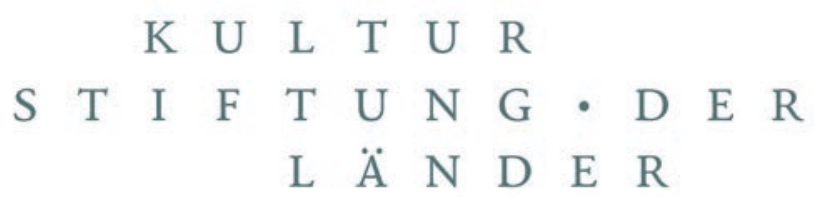

